\title{
Événements indésirables liés aux médicaments et pratique de la pharmacie hospitalière : Sortir de nos sentiers battus
}

par Peter J Zed

$\mathrm{U}$ n événement indésirable lié aux médicaments est la survenue d'un préjudice secondaire à l'emploi ou au mauvais emploi d'un médicament ${ }^{1}$. On estime que les événements indésirables liés aux médicaments sont responsables de plus de 17 millions de consultations aux services des urgences et de près de 9 millions d'hospitalisations par année aux États-Unis ${ }^{2,3}$. Un modèle de coût de la maladie publié en 2001 estimait les coûts annuels associés à la morbidité et à la mortalité secondaires aux événements indésirables liés aux médicaments à plus de 177 milliards de dollars américains ${ }^{3}$. Jusqu'à récemment, les effets de ces événements indésirables au Canada n'avaient pas encore été caractérisés, mais il est maintenant clair qu'on peut leur attribuer une morbidité, une mortalité et un fardeau économique considérables. Plus particulièrement, on a estimé que jusqu'à $25 \%$ des admissions en médecine générale et $12 \%$ des consultations des adultes aux services des urgences dans ce pays étaient directement associées aux événements indésirables liés aux médicaments, dont $70 \%$ pourraient être évités ${ }^{4,5}$. Dans l'étude canadienne sur les événements indésirables, on a observé que 7,5\% de tous les patients hospitalisés avaient subi un événement indésirable durant leur séjour à l'hôpital et que près de $24 \%$ des événements étaient associés à l'administration de médicaments ou de fluides $^{6}$. Enfin, dans une autre étude, $23 \%$ des patients avaient subi un événement indésirable dans les 30 jours suivant leur congé de l'hôpital, 72 \% des événements étaient liés aux médicaments et $50 \%$ des événements auraient pu être évités ${ }^{7}$. Ces données impressionnantes suggèrent que nous avons sousestimé l'ampleur de ce problème, mais elles nous offrent du même coup une occasion formidable d'examiner et de mettre en œuvre des stratégies permettant de réduire la survenue de ces événements chez les patients hospitalisés ou externes. Dans ce numéro du $J C P H$, trois articles nous rappellent encore une fois les conséquences des événements indésirables liés aux médicaments et des problèmes reliés à la pharmacothérapie chez les patients hospitalisés ${ }^{8-10}$.
Pendant plus de 20 ans, les soins pharmaceutiques ont été la structure sur laquelle reposaient nos modèles d'exercice de la pharmacie auprès des patients hospitalisés ${ }^{11}$. Laccès continu et prolongé aux patients, aux renseignements le concernant et à d'autres professionnels de la santé constitue pour les pharmaciens d'hôpitaux un milieu d'exercice unique, idéal pour reconnaître, résoudre et prévenir les problèmes reliés à la pharmacothérapie. En revanche, malgré les tentatives d'améliorer la communication aux acteurs communautaires des renseignements concernant le patient au moment de sa sortie de l'hôpital, il est clairement reconnu que cette transition ne se fait pas sans heurt. Des lacunes dans la communication, le suivi et la surveillance des soins mettent le patient à risque dans la période suivant immédiatement sa sortie de l'hôpital ${ }^{11,12}$. À plus long terme, le système d'éparpillement des fournisseurs de soins de santé dans le milieu communautaire crée des problèmes continus d'accès et de communication. Le pharmacien communautaire n'a qu'un accès limité aux renseignements concernant le patient et aux autres professionnels de la santé. La complexité de ces problèmes conjugués contribue aux taux élevés d'événements indésirables liés aux médicaments qu'on observe peu de temps après la sortie du patient de l'hôpital ${ }^{8}$ et à plus long terme, dans le milieu des soins ambulatoires ${ }^{13}$.

Il existe des exemples de programmes et de modèles de pratique qui tentent de résoudre ces problèmes. Toutefois, il y a quand même une occasion pour les pharmaciens hospitaliers, en collaboration avec d'autres professionnels de la santé, d'améliorer davantage les soins aux patients de retour dans la communauté. Il y a aussi une occasion pour les pharmaciens hospitaliers et les pharmaciens communautaires d'amorcer une collaboration plus étroite afin de prodiguer des soins pharmaceutiques véritablement axés sur le patient. Bien qu'ils aient une influence moindre sur les soins prodigués en milieu hospitalier, les changements apportés aux lois sur la pharmacie dans le pays ont permis aux pharmaciens communautaires d'élargir leur champ de pratique 
et de prodiguer des services de pharmacie plus complets. En outre, des modèles de rémunération appropriés sont présentement en développement dans certaines provinces afin d'appuyer les services de pharmacie élargis. Finalement, une utilisation accrue de la technologie contribuera à améliorer l'accès à l'information dans tous les milieux de pratique, facilitant ainsi une meilleure collaboration entre les professionnels de la santé des milieux hospitalier et extrahospitalier.

Nous devons commencer à sortir de nos sentiers battus de la pratique hospitalière, à explorer des modèles de pratique novateurs et à adopter des approches qui permettent de faire profiter de notre expertise les patients et les autres professionnels de la santé après la sortie des patients de l'hôpital. Les solutions ne sont pas simples, mais l'importance des conséquences des événements indésirables liés aux médicaments pour nos patients mérite que nous nous y attardions. Une collaboration plus étroite avec les professionnels de la santé du domaine communautaire qui prodiguent des soins à nos patients après leur hospitalisation est un bon départ. Je suis sûr qu'avec la prestation attentionnée, réfléchie et consentante de soins axés sur le patient, un modèle amélioré de soins en collaboration avec les professionnels de la santé du milieu communautaire aura un effet considérable sur la réduction des événements indésirables liés aux médicaments.

[Traduction par l'éditeur]

\section{References}

1. Nebeker JR, Barach P, Samore MH. Clarifying adverse drug events: a clinician's guide to terminology, documentation, and reporting. Ann Intern Med 2004;140(10):795-801.

2. Bates DW, Spell N, Cullen DJ, Burdick E, Laird N, Petersen LA, et al. Adverse Drug Events Prevention Study Group. The costs of adverse events in hospitalized patients. JAMA 1997;277(4):307-311.

3. Ernst FR, Grizzle AJ. Drug-related morbidity and mortality: updating the cost-of-illness model. J Am Pharm Assoc (Wash) 2001;41(2):192-199.

4. Samoy LJ, Zed PJ, Wilbur K, Balen RM, Abu-Laban RB, Roberts M. Drug-related hospitalizations in a tertiary care internal medicine service of a Canadian hospital: a prospective study. Pharmacotherapy 2006; 26(11):1578-1586
5. Zed PJ, Abu-Laban RB, Balen RM, Loewen PS, Hohl CM, Brubacher JR, et al. Incidence, severity and preventability of medication-related visits to the emergency department: a prospective study. CMAJ 2008;178(12): 1563-1569.

6. Baker GR, Norton PG, Flintoft V, Blais R, Brown A, Cox J, et al. The Canadian Adverse Events Study: the incidence of adverse events among hospital patients in Canada. CMAJ 2004;170(11):1678-1686.

7. Forster AJ, Clark HD, Menard A, Dupuis N, Chernish R, Chandok N, et al. Adverse events among medical patients after discharge from hospital. CMAJ 2004;170(3):345-349. Erratum dans : CMAJ 2004;170(5):771.

8. Cashin RP, Yang M. Medications prescribed and occurrence of falls in general medicine inpatients. Can J Hosp Pharm 2011;64(5):321-326.

9. Kanji Z, Su VCH, Mainra R. Nitrofurantoin-induced pulmonary reaction involving respiratory symptoms: case report. Can J Hosp Pharm 2011; 64(5):362-365.

10. Mabasa VH, Malyuk DL, Weatherby EM, Chan A. A standardized, structured approach to identifying drug-related problems in the intensive care unit: FASTHUG-MAIDENS. Can J Hosp Pharm 2011;64(5): 366-369.

11. van Walraven C, Taljaard M, Bell CM, Etchells E, Zarnke KB, Stiell IG, et al. Information exchange among physicians caring for the same patent in the community. CMAJ 2008;179(10):1013-1018.

12. Hepler CD, Strand LM. Opportunities and responsibilities in pharmaceutical care. Am J Hosp Pharm 1990;47(3):533-543.

13. Taché SV, Sönnichsen A, Ashcroft DM. Prevalence of adverse drug events in ambulatory care: a systematic review. Ann Pharmacother 2011;45(7-8): 977-989.

Peter J Zed, B. Sc., B. Sc. Pharm., ACPR, Pharm. D., FCSHP, est coordonnateur clinique, Département de pharmacie, et spécialiste de la pharmacothérapie, Médecine d'urgence, au Queen Elizabeth II Health Sciences Centre, et professeur agrégé, Collège de pharmacie et Département de médecine d'urgence, Université Dalhousie, Halifax, Nouvelle-Écosse. II est également rédacteur adjoint du JCPH.

\section{Adresse de correspondance :}

Dr Peter J Zed

Department of Pharmacy - Halifax Infirmary

Queen Elizabeth II Health Sciences Centre

1796 Summer Street

Halifax NS B3H 3A7

Courriel : peter.zed@dal.ca 